\title{
Predictores psicosociales de la exposición y difusión de noticias falsas en Costa Rica
}

\section{Psychosocial predictors of fake news exposure and diffusion in Costa Rica}

\section{Preditores psicossociais de exposição e disseminação de notícias falsas na Costa Rica}

Carlos Brenes Peralta, Universidad de Costa Rica, San José, Costa Rica (carlos.brenesperalta@ucr.ac.cr)

Rolando Pérez Sánchez, Universidad de Costa Rica, San José, Costa Rica (rolando.perez@ucr.ac.cr)

Ignacio Siles González, Universidad de Costa Rica, San José, Costa Rica (ignacio.siles@ucr.ac.cr)

RESUMEN|Este estudio investiga los predictores psicosociales y sociodemográficos de la exposición a noticias falsas entre adultos costarricenses, así como su autopercepción de la capacidad de reconocerlas y de difundirlas. Se condujo una encuesta representativa por muestreo estratificado aleatorio aplicada a 805 usuarios de teléfonos celulares en 2019 , con un $67 \%$ de mujeres y una edad promedio de 38,83 (DT=15,75). En elámbito actitudinal, los resultados muestran una asociación positiva entre el autoritarismo de derecha y la difusión intencional de noticias falsas en redes sociales. En elámbito motivacional, se encontraron asociaciones positivas entre una motivación defensiva y la exposición a noticias falsas en medios de comunicación y vía WhatsApp, así como asociaciones entre motivaciones defensivas y de precisión con la autopercepción de la capacidad de reconocer noticias falsas. Las mujeres, las personas con mayor nivel educativo y las más jóvenes se exponen más a noticias falsas, mientras que los hombres y las personas con mayor educación dicen ser más capaces de identificar noticias falsas.

PALABRAS CLAVE: noticias falsas; exposición; reconocimiento; difusión; motivaciones; actitudes; Costa Rica. 
ABSTRACT / This study examines the psychosocial and sociodemographic predictors of exposure, self-perceived capacity to recognize and diffusion offake news among Costa Rican adults. A survey was conducted in May 2019, with a representative sample of 805 Costa Rican adults owners of mobile phones, with $67 \%$ women and a mean age of $38.83(S D=15.75)$. A random stratified sampling method was used. From an attitudinal perspective, results show a positive association between right-wing authoritarianism and the intentional diffusion of fake news in social media. From a motivational perspective, positive associations were found between a defensive motivation and fake news exposure injournalistic media and via WhatsApp, as well as associations between defensive and accuracy motivations with the self-perceived capacity to recognize fake news. Finally, women, people with higher education, and those with younger age were more exposed to fake news, while men and people with higher education perceived themselves as more capable to identify fake news.

KEYWORDS: fake news; exposure; recognition; diffusion; motivations; attitudes; Costa Rica.

RESUMO | O estudo investiga os preditores psicossociais e sociodemográficos da exposição a notícias falsas entre adultos da Costa Rica, assim como sua autopercepção da capacidade de reconhecê-las e espalhá-las. Foi realizada uma pesquisa representativa com uma amostra estratificada aleatória aplicada a 805 usuários de celulares em 2019, com um 67\% de mulheres e de uma idade média de 38,83 (desvio-padrão=15,75). No âmbito atitudinal, os resultados mostram uma associação positiva entre o autoritarismo de direita e a disseminação intencional de notícias falsas em redes sociais. No âmbito motivacional, foram encontradas associações positivas entre uma motivação defensiva e a exposição a notícias falsas na mídia e via WhatsApp, bem como associações entre motivações defensivas e de precisão com a autopercepção da capacidade de reconhecer notícias falsas. As mulheres, as pessoas com mais nível educacional e as mais novas são mais expostas a notícias falsas, enquanto os homens e as pessoas com mais nível educacional dizem ser capazes de identificar notícias falsas.

PALAVRAS-CHAVE: notícias falsas; exposição; reconhecimento; disseminação; motivações; atitudes; Costa Rica. 


\section{INTRODUCCIÓN}

El fenómeno de las noticias falsas ha ganado creciente interés académico y sociopolítico. La investigación y la discusión pública sobre el tema se centran en los posibles efectos de su difusión en la cultura política y en el devenir democrático, así como en los mecanismos sociocognitivos y comunicativos que lo posibilitan (Tong et al., 2020; Valenzuela, et al., 2021; Waisbord, 2018). En Costa Rica, las noticias falsas han jugado un rol preponderante, por su vínculo con eventos mediáticos particulares (Siles et al., 2021). La preocupación por la interacción de los costarricenses con contenidos falsos en Facebook se vincula con tres eventos recientes: 1) protestas ciudadanas en 2019 que provocaron la renuncia del Ministro de Educación Pública por una serie de políticas implementadas, 2) la discusión en el plenario legislativo sobre la importancia de las noticias falsas como problema democrático (2019 y 2020), y 3) la emergencia sanitaria por el coronavirus desde marzo 2020 (Carazo et al., 2020). Las noticias falsas también fueron objeto de atención pública en Costa Rica cuando sirvieron como insumo para una marcha xenofóbica contra nicaragüenses a mediados de 2018 (Carazo et al., 2020).

Las primeras unidades de verificación de datos en Costa Rica fueron creadas en 2018. Medios de comunicación impresos fundaron No Coma Cuento (La Nación) y No se Vaya Pollo (La Teja) en enero de 2018, de cara al proceso electoral de ese año. Pasadas las elecciones presidenciales, surgieron unidades como No Caiga (CRHoy. com) y Doble Check (Universidad de Costa Rica). En 2019, las instituciones públicas establecieron nuevas iniciativas para contrarrestar la difusión de noticias falsas: la Presidencia de la República de Costa Rica lanzó la plataforma Gobierno Aclara y el Tribunal Supremo de Elecciones estableció acuerdos con Facebook y Twitter en materia de alfabetización ciudadana.

En ese contexto, este estudio considera tres aspectos asociados con las noticias falsas: la frecuencia con que las personas identifican noticias que no son totalmente verdaderas en medios de comunicación, redes sociales y WhatsApp, la autopercepción sobre la capacidad de reconocer noticias falsas, y si las personas comparten noticias que consideraban falsas en redes sociales y WhatsApp. Específicamente, se analizan los predictores psicosociales y sociodemográficos de la exposición, autopercepción de la capacidad de reconocimiento y difusión de noticias falsas entre adultos costarricenses.

\section{LAS NOTICIAS FALSAS COMO OBJETO DE ESTUDIO}

Un importante debate académico se ha centrado en definir una noticia falsa. Se pueden encontrar hasta doce definiciones distintas. Egelhofer y Lecheler (2019) integraron las distintas definiciones y proponen una conceptualización a partir 
de dos dimensiones. Primero, el género de las noticias falsas, que consiste en crear deliberadamente desinformación pseudo-periodística, y en segundo lugar, la etiqueta de las noticias falsas, la instrumentalización del término para deslegitimar a los medios de comunicación. Este manuscrito trabaja desde la primera dimensión.

El género de las noticias falsas se distingue por tres características, que lo distinguen de falsedades, periodismo de baja calidad o errores de comunicación. Una noticia debe considerarse como falsa si, primero, posee un bajo nivel de facticidad. Es decir, aunque una noticia falsa tenga información correcta, la mayoría de su contenido es fabricado o falso (Tandoc et al., 2018). Una segunda característica es que imitan la forma del contenido de las noticias periodísticas. Como resultado, las audiencias pueden confundir las noticias falsas como genuinas y creíbles (Mustafaraj \& Metaxas, 2017). Una tercera característica es que son creadas desde motivaciones políticas, ideológicas o financieras para engañar deliberadamente a una audiencia (Tandoc et al., 2018) (ver gráfico 1).

Las noticias falsas se discuten comúnmente en el contexto del estudio de la desinformación y de la información errónea. Mientras la desinformación consiste en información incorrecta o sesgada que se difunde deliberadamente, la información errónea refiere a información incorrecta o sesgada difundida de manera no intencional (Lazer et al., 2018). Por tanto, el concepto de las noticias falsas se ubica mejor dentro de la literatura sobre la desinformación. Conceptos como la desinformación, los rumores, las teorías de conspiración y la propaganda pueden clasificarse como noticias falsas si cumplen las tres características mencionadas (Egelhofer \& Lecheler, 2019).

La investigación sobre noticias falsas ha girado alrededor de dos grandes ejes: exposición y difusión. El primero se interesa en entender cuestiones de exposición, es decir, "la medida en que miembros de la audiencia han encontrado mensajes o clases de mensajes/contenidos específicos de los medios de comunicación" (Slater, 2004, p. 168). En el caso de las noticias falsas, la exposición se refiere a las dinámicas involucradas en la recepción de noticias percibidas como falsas (Tandoc, Ling et al., 2018; Wagner \& Boczkowski, 2019). Se privilegia la forma en que las personas se relacionan con el contenido catalogado como falso, cómo lo reciben y cómo lo interpretan. Estas investigaciones han demostrado que la aceptación de noticias falsas varía entre personas. Por ejemplo, la creencia en noticias falsas se ha asociado con el dogmatismo y el fundamentalismo religioso (Bronstein et al., 2019). Para De keersmaecker y Roets (2017), la efectividad de estrategias correctivas para contrarrestar los efectos negativos de las noticias falsas en factores cognitivos es mayor en personas con habilidades cognitivas más avanzadas. 


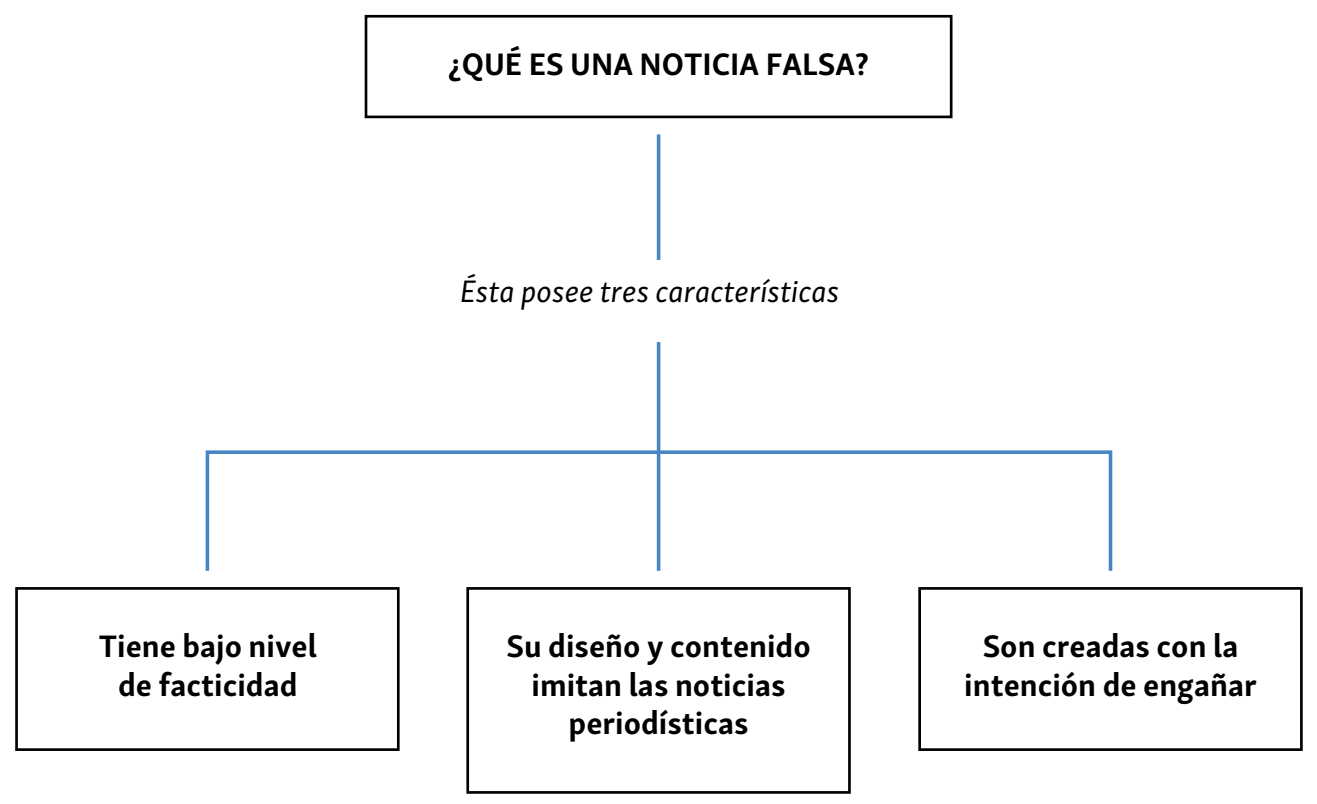

Gráfico 1. Características definitorias de las noticias falsas

Fuente: Elaboración propia.

Un segundo eje de investigación busca entender las dinámicas de difusión de noticias falsas y el alcance entre diferentes segmentos de la población. Sitúan las noticias falsas en un contexto más amplio de producción, circulación y consumo (Hoffman et al., 2019). Según estos estudios, un primer factor que condiciona la difusión es el acceso a tecnologías específicas. Estos trabajos se centran en el rol de los algoritmos en la forma en que ciertos contenidos adquieren visibilidad en algunos grupos sociales (Diakopoulos, 2019). Investigaciones más recientes destacan la creciente importancia de WhatsApp como vehículo para difundir noticias falsas, dado su uso creciente como medio informativo (Newman et al., 2019; Valenzuela et al., 2021). También concluyen que los factores psicosociales condicionan la difusión de noticias falsas.

Se considera central en este estudio evaluar el rol que juega el conservadurismo y sus correlatos psicosociales en reconocer y difundir noticias falsas. Estudios sobre los procesos electorales de Donald Trump en Estados Unidos y Jair Bolsonaro en Brasil asocian las actitudes y discursos vinculados al conservadurismo con difundir noticias falsas (Van der Linden et al., 2020; Ituassu et al., 2019; Da Silva $\&$ Larkins, 2019). Otros autores han encontrado este correlato en un marco no electoral (Guess et al., 2019), por lo que su estudio a nivel local permite aportar a la comprensión de la génesis de la difusión de este tipo de noticias en Costa Rica. 


\section{PREDICTORES PSICOSOCIALES Y SOCIODEMOGRÁFICOS DE LA EXPOSICIÓN Y DIFUSIÓN DE NOTICIAS FALSAS}

Esta investigación asocia variables psicosociales -los factores de personalidad y lo mecanismos cognitivos- con los tres aspectos sobre noticias falsas. El primer ámbito examina aspectos de la personalidad, específicamente actitudes conservadoras o que legitiman actitudes autoritarias y de jerarquización social para aceptar noticias falsas. El segundo ámbito, motivacional, se vincula con mecanismos cognitivos que favorecen la aceptación y la reproducción de noticias falsas, donde lo que importa es estar informado y evaluar la información recibida.

\section{Autoritarismo, dominancia social y noticias falsas}

El autoritarismo se entiende como producto de la obediencia irrestricta a las autoridades (sumisión autoritaria), recurrir al castigo como medio legítimo de control social (agresión autoritaria), y respaldar un sistema tradicional de normas (convencionalismo) (Altemeyer, 1981, 1996, basado en la teoría de la personalidad autoritaria de Adorno et al., 1950). Así, las personas autoritarias abogan por el castigo durante la infancia, deploran la indulgencia en los tribunales y creen que las reformas penales solo animan a los criminales a continuar cometiendo actos ilegales (Altemeyer, 1996).

La dominancia social considera que la organización social humana tiende a estructurarse jerárquicamente. Implica que un grupo se ubica en una posición hegemónica, con un valor intrínseco al poseer poder político, social y un acceso privilegiado a recursos socioeconómicos y al bienestar social (Pratto et al., 1994). Estos grupos construyen discursos sociales e históricos que les permiten legitimar las asimetrías sociales y justificar lo que ellos consideran grupos inferiores (Sidanius et al., 2004).

Los constructos autoritarismo de derecha y dominancia social están relacionados, pero no son sinónimos. La dominancia social refiere al respaldo de actitudes antidemocráticas que legitiman la desigualdad estructural. En cambio, el autoritarismo se concentra en defender valores asociados a las sociedades tradicionales, en donde la religión juega un rol central para otorgar sentido a dichos valores (Bilewicz et al., (2017); Crowson \& Brandes (2017); Pérez Sánchez et al., (2020).

Según Pérez Sánchez et al., (2020), la presencia de actitudes que respaldan el autoritarismo y la dominancia social en las sociedades actuales se asocia con una insatisfacción hacia la política, lo cual implica un rechazo generalizado al actuar de los políticos, al valorar que no están cumpliendo con su rol. Esta insatisfacción nombrada por los autores como animadversión hacia la política puede variar, según 
las políticas de gobierno, el estado de la economía y el actuar de las instituciones estatales (Montero et al., 2008).

Los antecedentes sobre la relación de estos factores con las noticias falsas han mostrado que las personas con mayores puntajes de autoritarismo consumen menos diversidad de fuentes de noticias (Sindermann et al., 2020). Asimismo, altos puntajes de autoritarismo y dominancia social se asocian con una mayor tolerancia a la difusión de contenido falso por parte de políticos ideológicamente cercanos (De keersmaecker \& Roets, 2019).

\section{La dimensión motivacional de la exposición y procesamiento de noticias falsas}

La teoría del razonamiento motivado ha sido importante para comprender cómo la selección y el procesamiento de contenido noticioso varía según las motivaciones de las personas (Taber \& Lodge, 2006; Winter et al., 2016). Esta teoría propone que las motivaciones individuales influyen en los procesos cognitivos que utilizan las personas para seleccionar y procesar información (Kunda, 1990). La motivación se define como "cualquier deseo, anhelo o preferencia que involucra el resultado de una tarea de razonamiento" (Kunda, 1990, p. 480). Hay dos motivaciones que se consideran centrales: la defensiva y la de precisión (Leeper \& Slothuus, 2014).

Los individuos motivados defensivamente seleccionan y procesan información para validar y proteger sus actitudes, creencias y comportamientos existentes (Kunda, 1990). Prefieren y valoran más positivamente la información que refuerza sus conclusiones deseadas, mientras que desacreditan información discordante. En cambio, las personas motivadas por precisión utilizan estrategias cognitivas para llegar a una conclusión sobre un tema específico. Procesan la información de manera objetiva, independientemente de si refuerza sus propias creencias y actitudes.

La evidencia sobre ambas motivaciones proviene mayoritariamente de experimentos. Estos estudios se han complementado con mediciones correlacionales, que utilizan encuestas para capturar las dimensiones conceptuales de las motivaciones (Federico \& Schneider, 2007). Varios estudios han sugerido que, a nivel de diferencias individuales, need for cognition equivale a una manipulación experimental de la motivación de precisión (Nir, 2011; Cacioppo et al., 1996). Esta necesidad se define como la tendencia crónica de las personas hacia el disfrute de la actividad cognitiva que implique un esfuerzo (Cacioppo \& Petty 1982; Cacioppo et al., 1996).

En cambio, la definición operacional de la motivación defensiva puede capturarse mediante need to evaluate ( $\mathrm{Nir}, 2011)$, la tendencia crónica de las personas a formar juicios y pensamientos evaluativos (Jarvis et al., 1996). Need to evaluate se ha asociado positivamente con una evaluación más intensa de candidatos políticos (Bizer et 
al., 2002, 2004), con un mayor compromiso partisano y una menor ambivalencia política (Federico \& Schneider 2007).

Varios autores han aplicado la teoría del razonamiento motivado al contexto de las noticias falsas. Argumentan que la motivación defensiva es un factor importante para entender por qué las personas le prestan atención y creen que las noticias falsas de tipo pro-actitudinal son veraces (Beck, 2019). Además, se ha sugerido que las noticias falsas influyen en adquirir o reforzar ideas falsas sobre diversos temas y que algunas personas son resistentes a correcciones explícitas de estas ideas, debido a la influencia de sesgos cognitivos en el procesamiento de la información que activa una motivación defensiva (Berinsky, 2017). Esto explicaría por qué el fact-checking solamente tendría un efecto cuando la corrección de ideas falsas no contradice la conclusión deseada de una persona (Hameleers $\&$ Van der Meer, 2019). En cambio, otros estudios han encontrado que personas entrenadas en estrategias de pedagogía de medios, son influenciadas más por una motivación de precisión -que por una motivación defensiva- al procesar noticias falsas (Kahne \& Bowyer, 2017).

No se encontraron investigaciones que examinen la relación entre need to evaluate -como proxy conceptual de una motivación defensiva- y de need for cognition-como proxy de una motivación de precisión- con las noticias falsas. No obstante, se considera relevante estudiar la relación entre ambos constructos con los tres aspectos de estudio sobre noticias falsas, dada la aplicabilidad de la teoría de razonamiento motivado para explicar la dimensión motivacional de la exposición, procesamiento y difusión de contenido noticioso y falso, así como la utilidad de need for cognition y de need to evaluate para operacionalizar el razonamiento motivado en estudios correlacionales.

\section{Características sociodemográficas en el estudio de las noticias falsas}

Para contribuir a comprender la aceptación y difusión de noticias falsas, junto con las dimensiones psicosociales, es relevante considerar variables sociodemográficas, en particular la edad, el sexo, la zona geográfica de residencia y el nivel educativo. La edad y la zona geográfica importan, al referir a formas de uso y acceso al contenido noticioso; se asocian a las diferencias generacionales vinculadas con el empleo de las tecnologías digitales, así como con las brechas prevalecientes que limitan el acceso y uso en las zonas rurales. El nivel educativo puede afectar en el tipo de contenido consumido, las fuentes informativas y su evaluación. Con respecto al sexo, es necesario indagar si existen diferencias a nivel actitudinal, o en los factores motivacionales que favorezcan o limiten la aceptación y difusión de noticias falsas. 
Rampersad y Althiyabi (2020) encontraron que la edad, más que la educación o el sexo, explican la aceptación de noticias falsas. En lo referente a la educación, determinaron que un mayor nivel educativo se asocia con una menor aceptación de noticias falsas.

\section{METODOLOGÍA}

\section{Preguntas de investigación}

Las preguntas orientadoras de este estudio fueron las siguientes:

P1. ¿Cuál es la asociación existente entre la exposición, la autopercepción sobre la capacidad de reconocer y difundir noticias falsas y el respaldo de actitudes conservadoras, expresadas en altos puntajes de autoritarismo y dominancia social?

P2. ¿Cuál es la asociación entre los tres aspectos sobre noticias falsas y los factores motivacionales de consumo de noticias asociados a need to evaluate y need for cognition?

P3. ¿Cuál es la relación entre los tres aspectos sobre noticias falsas y la edad, género, nivel educativo y la zona geográfica de residencia de las personas?

\section{Participantes}

Se reclutaron 805 personas de todo el país, en el marco de la Encuesta Nacional de Hogares de 2017 que abarca 97\% de la población mayor de 18 años en Costa Rica. Se recurrió a un muestreo estratificado aleatorio, tomando como marco muestral el Plan Nacional de Numeración de la Superintendencia de Telecomunicaciones (SUTEL). Las personas se contactaron directamente a su teléfono celular, solicitando su disposición a participar en el estudio. Como criterios de inclusión, se entrevistaron solamente personas mayores de 18 años y con nacionalidad costarricense. La recolección se llevó a cabo del 13 al 19 de mayo de 2019, mediante entrevistas telefónicas realizadas en horarios de mañana, tarde y noche. El 67\% de la muestra son mujeres, con una edad que oscila entre los 18 y 88 años $(M=38,83$, DT=15,75). Un $42 \%$ de las personas tienen educación primaria, 37\%, educación secundaria y $21 \%$, educación universitaria. Considerando un nivel de confianza de $95 \%$, se estima un error muestral de $\pm 3,45$ puntos porcentuales.

A cada persona encuestada se le indicó que su participación era voluntaria y el tratamiento de los datos, confidencial y anónimo. Aclarado esto, se le solicitó su acuerdo verbal para participar en la encuesta. Durante esta, se mantuvo control de las cuotas correspondientes a la distribución nacional por provincia, sexo y 
edad (Instituto Nacional de Estadística y Censos, 2017). Se ponderó la variable educación para equilibrar los niveles de primaria y secundaria.

\section{Instrumentos}

Los cinco instrumentos aplicados utilizaron escalas tipo Likert de 5 puntos $(1=$ totalmente en desacuerdo, 5 = totalmente de acuerdo). Para medir el autoritarismo, se recurrió a la versión corta de Escala de Autoritarismo de Derecha de Altemeyer (1996) de seis ítems. Un ejemplo de ítem es "Las leyes de dios sobre la pornografía, el aborto y el matrimonio deben ser seguidas estrictamente, sus transgresiones deben ser castigadas". Se obtuvo un Alfa de Cronbach de ,79.

Para estudiar la legitimación de las jerarquías sociales y la desigualdad, se empleó la Escala de la de Dominancia Social (Sidanius \& Pratto,1999), utilizando una versión reducida de ocho ítems, compuesta por dos subescalas: cuatro ítems que miden el respaldo a las jerarquías sociales y cuatro actitudes a favor de la igualdad social. Un ejemplo de losítems correspondientes al respaldo a las jerarquías es: "Para salir adelante en la vida, algunas veces es necesario pasar por encima de las otras personas". Para las actitudes a favor de la igualdad: “Tendríamos menos problemas si tratáramos a los diferentes grupos sociales más equitativamente". Se obtuvo un coeficiente de consistencia interna de ,66 (jerarquización social) y ,68 (igualdad).

Para medir el descontento hacia la política y los políticos, se recurrió a la Escala de la animadversión hacia los políticos (Pérez Sánchez et al., 2020) , compuesta por 10 ítems en una escala Likert de 5 puntos. Un ejemplo de los ítems es: "Los políticos lo único que quieren es sacar provecho personal al ser nombrados en el gobierno". Se obtuvo un coeficiente de Cronbach de ,91.

Para estudiar need for cognition, se utilizó la versión corta de dos ítems desarrollada por el American National Election Study (2013). Los dos ítems son: "Yo prefiero resolver problemas complejos en vez de problemas simples"; "Me gusta tener la responsabilidad de manejar una situación que requiere pensar mucho." La medida obtuvo una consistencia interna de, 54 .

Se utilizó la escala reducida de tres ítems para medir need to evaluate (Bizer et al., 2004). Los tres ítems son: "Tengo una opinión sobre casi todo"; "Para mí es importante tener una opinión acerca de si algo es bueno o malo"; "Es muy importante para mí tener una opinión fuerte". Se obtuvo un alfa de Cronbach de ,59.

Para medir la exposición a noticias falsas, se formuló una pregunta que indaga sobre la frecuencia de exposición a noticias falsas en medios de comunicación, redes sociales y WhatsApp. Se empleó una escala de respuesta de 5 puntos, que va de nunca (1) a siempre (5). 
Para estudiar la autopercepción sobre la capacidad de reconocer noticias falsas, se formuló una pregunta que indaga sobre la frecuencia con la que la persona logra identificar noticias que considera que no son totalmente verdaderas, en medios de comunicación, redes sociales y WhatsApp. Igualmente, se empleó una escala de respuesta de 5 puntos, que va de nunca (1) a siempre (5).

Se indagó si las personas compartieron noticias que consideraban falsas. Para ello, se consultó directamente si se habían compartido noticias de este tipo en redes sociales o por WhatsApp. Se utilizó una respuesta dicotómica, sí/no.

Como variables sociodemográficas se consideraron la edad, el nivel educativo, el sexo y la provincia de residencia. Para fines analíticos, la edad se recodificó en tres grupos - 18 a 34 años, 35 a 54 años y 55 años y más-. La medida de educación se recodificó en tres grupos -educación primaria, secundaria y universitaria-. La variable con las siete provincias costarricenses de residencia se recodificó en una dicotómica: Valle Central, que incluye vivir en las provincias de San José, Heredia, Alajuela y Cartago. La categoría fuera del Valle Central comprende las provincias costeras de Puntarenas, Limón y Guanacaste.

Como procedimiento de análisis se realizaron análisis descriptivos básicos generales y análisis de factores exploratorios. Además, se estimaron varios modelos de regresión jerárquica y de regresión logística utilizando el método Stepwise, para conocer el impacto relativo de las medidas psicosociales y sociodemográficas sobre la exposición, autopercepción sobre la capacidad de reconocimiento y difusión de noticias falsas.

\section{RESULTADOS}

\section{Análisis descriptivos básicos}

Para comenzar, se examinaron las tendencias a la exposición a noticias falsas. Se preguntó sobre la frecuencia con la que las personas tienden a encontrar noticias que no son completamente verdaderas en medios de comunicación, redes sociales y vía WhatsApp. En promedio, en una escala de 1 a 5 , las personas encuestadas encontraron noticias falsas con igual frecuencia en las redes sociales $(M=3,02 ; D T=1,18)$ y en los medios de comunicación $(M=2,96 ; D T=1,12)$. Este promedio es similar al de la frecuencia de exposición a noticias falsas vía WhatsApp ( $M=2,90 ; \mathrm{DT}=1,25)$.

También se preguntó si las personas compartieron noticias que consideraban falsas. En el caso de redes sociales, 81\% afirmó no haberlo hecho y 19\%, sí. El número es casi idéntico en el caso de WhatsApp: 18\% compartió noticias falsas y $82 \%$ afirmó que no. 
Finalmente, $36 \%$ de la muestra afirmó tener mucha o demasiada capacidad para reconocer las noticias falsas, 33\% dijo ser algo capaz y 30\% consideró que tiene poca o ninguna capacidad.

\section{Predictores de la exposición, autopercepción sobre capacidad de reconocimiento y difusión de noticias falsas}

Para conocer el impacto relativo de las medidas psicosociales sobre la exposición y autopercepción sobre la capacidad de reconocer noticias falsas, considerando la edad, el sexo, el nivel educativo y la región geográfica como variables intervinientes, se calcularon cuatro modelos de regresión jerárquico utilizando el método Stepwise. Se estimó un modelo jerárquico para cada variable dependiente: la exposición a noticias falsas en medios de comunicación, la exposición en redes sociales, la exposición vía WhatsApp y la autopercepción sobre la capacidad de reconocer noticias falsas. Se utilizaron los mismos predictores en cada regresión jerárquica. En el primer nivel de regresión se incluyeron need for cognition y need to evaluate. En el segundo nivel se incorporaron autoritarismo, animadversión hacia los políticos, dominancia social e igualitarismo. En el tercer nivel se incluyeron el nivel educativo, la edad, la región geográfica y el sexo. Los resultados completos de los modelos de regresión se presentan en la tabla 1. Por motivos de espacio, se describen a continuación los resultados del tercer nivel jerárquico de cada regresión.

La exposición a noticias falsas en medios de comunicación se asoció con altos puntajes en need to evaluate $(\beta=, 09, p<, 05)$ y un nivel educativo universitario $(\beta=42, p<, 01)$-en comparación con estudios primarios, $R^{2}(\mathrm{~N}=707)=, 05$, $F(13,694)=2,73, p<, 01)$.

Por su parte, una mayor frecuencia de exposición a noticias falsas en redes sociales se asoció con personas con estudios universitarios -en comparación con aquellas con educación primaria $(\beta=, 32, p<, 01)$-, a personas con edades entre 18 a 24 años -en comparación con las de 55 años o más $(\beta=-, 28, p<, 01)$, y las de sexo femenino $\left.(\beta=, 21, p<, 01), R^{2}(\mathrm{~N}=693)=, 12, F(14,679)=6,39, p<, 01\right)$.

La exposición a noticias falsas vía WhatsApp es mayor entre personas con altos puntajes en need to evaluate $(\beta=, 10, p<, 05)$, bajos puntajes en dominancia social ( $\beta=-$ $, 10, p<, 05)$ y aquellas con educación universitaria -en comparación con aquellas con nivel primario $(\beta=, 57, p<, 01), R^{2}(\mathrm{~N}=696)=, 32, F(13,683)=5,82, p<, 01$.

Una mayor autopercepción sobre la capacidad para reconocer noticias falsas se asoció con el sexo masculino $(\beta=-33, p<, 01)$, con niveles de educación secundaria $(\beta=, 34, p<, 01)$ y universitaria $(\beta=, 52, p<, 01)$, así como con altos puntajes en need for cognition $(\beta=, 09, p<, 05)$ y need to evaluate $(\beta=, 09, p<, 05), R^{2}(N=706)=, 36$, $F(13,693)=7,77, p<, 01)$. 


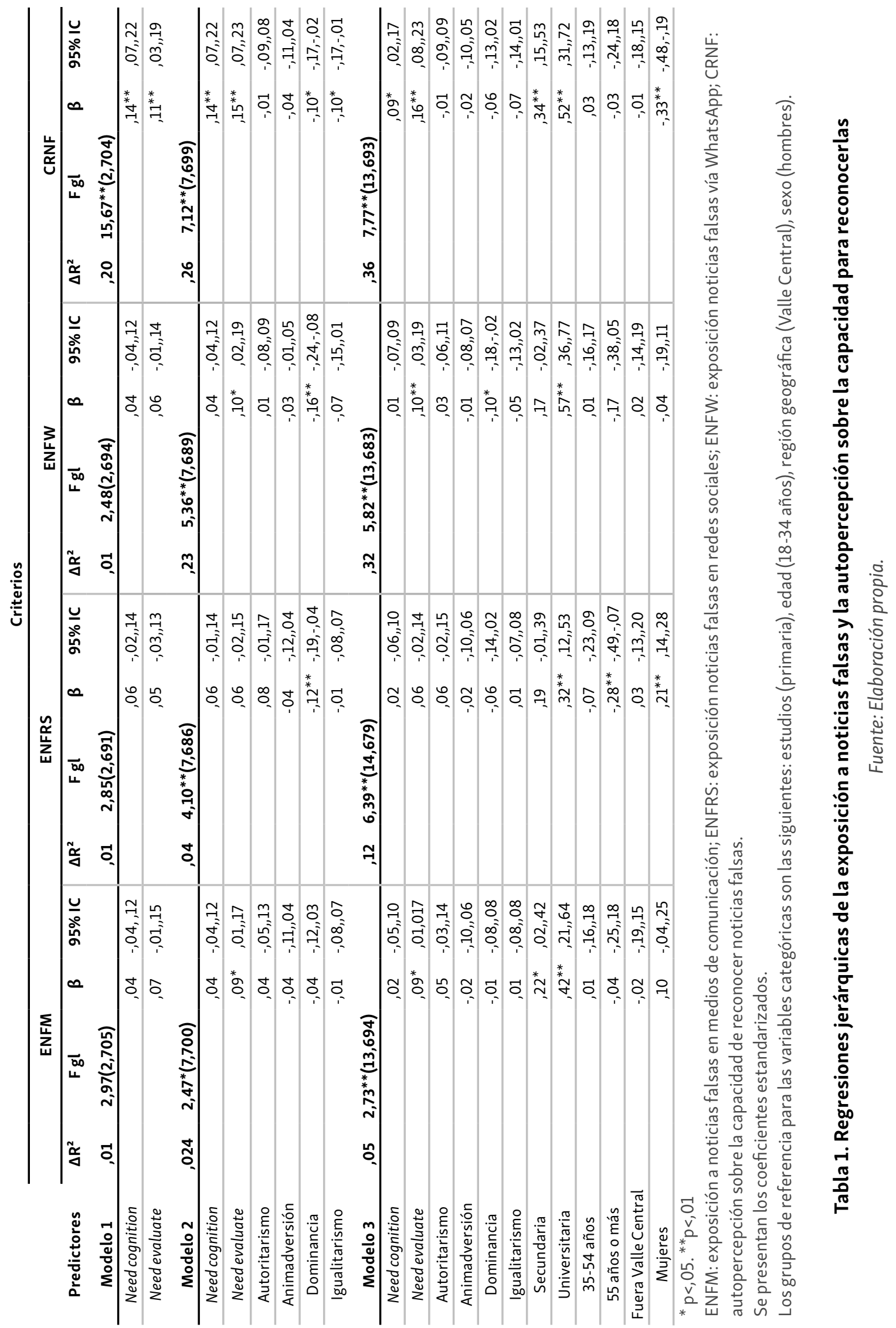


Criterios

\begin{tabular}{|c|c|c|c|c|c|c|c|c|}
\hline \multirow[b]{2}{*}{ Predictores } & \multicolumn{4}{|c|}{ CNFRS } & \multicolumn{4}{|c|}{ CNFW } \\
\hline & $\Delta \mathrm{R}^{2} \mathrm{McF}$ & $X^{2} g l$ & RM & $95 \%$ IC & $\Delta \mathrm{R}^{2} \mathrm{McF}$ & $X^{2} g l$ & RM & $95 \%$ IC \\
\hline Modelo 1 & ,001 & $1,58(2)$ & & & ,001 &, $82(2)$ & & \\
\hline Need cognition & & & 1,06 & ,96,1,18 & & & 1,05 & ,95,1,16 \\
\hline Need evaluate & & & ,95 & $, 84,1,08$ & & & ,98 & $, 86,1,12$ \\
\hline Modelo 2 &, 02 & $12,94(7)$ & & & 01 & $7,47(7)$ & & \\
\hline Need cognition & & & 1,06 & $96,1,18$ & & & 1,05 & $94,1,16$ \\
\hline Need evaluate & & & 89 & ,78,1,02 & & & 95 & ,83,1,09 \\
\hline Autoritarismo & & & $1,20^{* *}$ & $1,05,1,38$ & & & $1,16^{*}$ & $1,01,1,33$ \\
\hline Animadversión & & & 1,05 & $94,1,17$ & & & 1,02 & ,91,1,14 \\
\hline Dominancia & & & 93 & $, 84,1,03$ & & & 1,02 & $92,1,12$ \\
\hline Igualitarismo & & & 99 & $, 87,1,13$ & & & 96 & $, 85,1,09$ \\
\hline Modelo 3 & ,03 & $20,40(14)$ & & & ,02 & $14,16(13)$ & & \\
\hline Need cognition & & & 1,05 & $95,1,17$ & & & 1,03 & $92,1,14$ \\
\hline Need evaluate & & & 90 & $, 78,1,03$ & & & 96 & $, 84,1,10$ \\
\hline Autoritarismo & & & $1,17^{*}$ & $1,01,1,35$ & & & 1,14 & $99,1,31$ \\
\hline Animadversión & & & 1,04 & $93,1,17$ & & & 1,01 & $90,1,13$ \\
\hline Dominancia & & & 94 & $84,1,04$ & & & 1,02 & $92,1,14$ \\
\hline Igualitarismo & & & 99 & $, 87,1,13$ & & & 96 & ,85,1,09 \\
\hline Secundaria & & & 1,43 & $, 84,2,44$ & & & 1,69 & $98,2,90$ \\
\hline Universitaria & & & 1,03 & $, 58,1,84$ & & & 1,33 & ,74,2,39 \\
\hline 35-54 años & & & 1,11 & $, 72,1,69$ & & & 1,01 & ,66,1,55 \\
\hline 55 años o más & & & 90 & $, 50,1,62$ & & & 94 & $, 53,1,67$ \\
\hline Fuera Valle Central & & & ,98 & $, 64,1,51$ & & & 1,26 & , 82,1,92 \\
\hline Mujeres & & & 99 & 67,1,45, & & & ,82 & ,56,1,21 \\
\hline
\end{tabular}

$\mathrm{p}<, 05 .{ }^{*} \mathrm{p}<, 01$

CNFRS: compartir noticias falsas en redes sociales; CNFW: compartir noticias falsas vía WhatsApp.

RM: Razón de probabilidades.

Los grupos de referencia para las variables categóricas son las siguientes: estudios (primaria), edad (18-34 años),

región geográfica (Valle Central), sexo (hombres).

Tabla 2. Regresiones logísticas de la difusión de noticias falsas en redes sociales y vía WhatsApp

Fuente: Elaboración propia. 
Para estudiar el impacto relativo de las mismas mediciones psicosociales y sociodemográficas sobre la difusión de noticias falsas en redes sociales y vía WhatsApp, se calcularon dos modelos de regresión logística utilizando el método Stepwise. En cada modelo, los tres niveles de regresión fueron los mismos utilizados en las regresiones jerárquicas. Para facilitar la interpretación de los coeficientes significativos, se calculó la probabilidad de compartir noticias falsas a partir de la razón de probabilidades (ver tabla 2).

El modelo logístico para predecir la difusión de noticias falsas en redes sociales resultó no significativo en los tres niveles. Sin embargo, a nivel univariado y al controlar por el impacto de las variables sociodemográficas en el tercer nivel, la probabilidad de difundir noticias falsas en redes sociales se mantuvo en $54 \%$ para las personas con altos puntajes en autoritarismo $(\mathrm{RM}=1,17, p<, 05)$.

De igual manera, los tres niveles del modelo para predecir la difusión de noticias falsas vía WhatsApp resultaron no significativos. En el segundo modelo, puntajes altos en autoritarismo se asociaron con una probabilidad de $54 \%$ de compartir noticias falsas vía WhatsApp ( $\mathrm{RM}=1,16, p<, 05)$. Sin embargo, el autoritarismo no se mantuvo como predictor de la variable dependiente en el tercer nivel.

\section{DISCUSIÓN}

Los resultados reflejan una asociación positiva entre el autoritarismo de derecha y la difusión intencional de noticias falsas en redes sociales. A nivel motivacional, existen asociaciones positivas entre una motivación defensiva y la exposición a noticias falsas en medios de comunicación y WhatsApp, así como asociaciones entre motivaciones defensivas y de precisión con la autopercepción de la capacidad de reconocer noticias falsas. A nivel sociodemográfico, mujeres, personas con mayor nivel educativo y aquellas más jóvenes afirmaron estar más expuestas a noticias falsas, mientras que los hombres y las personas con mayor educación afirman ser más capaces de identificar noticias falsas.

Este estudio examinó el rol de las actitudes conservadoras y de los factores motivacionales, controlando por características sociodemográficas, en la predicción de la exposición, la autopercepción sobre la capacidad de reconocimiento y la difusión de noticias falsas, en una muestra representativa de adultos costarricenses.

La primera pregunta indagó en la asociación entre las tres mediciones sobre noticias falsas y el respaldo a actitudes conservadoras, expresadas en altos puntajes de autoritarismo y dominancia social. En respuesta a esta pregunta, el hallazgo más importante es que el autoritarismo es relevante para entender la tendencia a difundir noticias falsas de manera intencional. De manera hipotética, el resultado 
posiblemente se deba a que la información que se difunde intencionalmente es pro-actitudinal y le permite defender o afirmar un proyecto de sociedad o atacar perspectivas que amenazan sus concepciones de mundo. Al respecto, De keersmaecker y Roets (2019) señalan que las personas más conservadoras están más dispuestas a tolerar la difusión de noticias falsas por parte de personajes de la política que son afines ideológicamente. Dada la relevancia del resultado, es necesario realizar mayor investigación al respecto.

La segunda pregunta del estudio indagó en la asociación entre las mediciones sobre noticias falsas y los constructos de need to evaluate y need for cognition considerados como proxis de una motivación defensiva y de una motivación hacia la precisión, respectivamente-. Un hallazgo relevante es que una motivación defensiva se asocia positivamente con exposición a noticias falsas en medios de comunicación y vía WhatsApp, pero no así en redes sociales.

La literatura ofrece suficiente evidencia para sugerir por qué la motivación defensiva se asocia a una mayor exposición a noticias falsas en medios de comunicación. Las personas exhiben dos tipos de sesgos cognitivos en la selección y procesamiento de noticias: uno de congruencia actitudinal, de manera que los mensajes pro-actitudinales son evaluados como más creíbles que los contraactitudinales, y un sesgo de desconfirmación, según el cual las personas evalúan positivamente los mensajes pro-actitudinales y denigran los contra-actitudinales (Taber et al., 2009). Ambos sesgos son las estrategias prevalentes utilizadas por las personas motivadas defensivamente al exponerse a las noticias (Taber \& Lodge, 2006). En este estudio es posible ver que las personas motivadas defensivamente utilizan la etiqueta de noticias falsas (Egelhofer \& Lecheler, 2019) como estrategia para deslegitimar las noticias de medios de comunicación de tipo contra-actitudinal. Esta perspectiva podría ser respaldada desde el muy documentado hostile media effect (Hansen \& Kim, 2011).

Por su parte, la asociación positiva entre motivación defensiva y exposición a noticias falsas vía WhatsApp podría tener varias explicaciones. Primero, en este espacio proliferan noticias falsas y las personas pueden percibir o exponerse con facilidad a falsedades en este contexto. Segundo, las personas pueden desconfiar de la veracidad tanto de las fuentes autoras de una noticia falsa como de ciertas personas que las comparten. Tercero, las personas motivadas defensivamente pueden aplicar la etiqueta de noticia falsa a noticias contra-actitudinales que encuentran en WhatsApp, pero no así a las pro-actitudinales.

Es interesante que la motivación defensiva no se asoció con una exposición a noticias falsas en redes sociales. Antes de proponer interpretaciones sobre este hallazgo, sería relevante estudiar la diversidad de las dietas informativas de las 
y los costarricenses en redes sociales, su relación con los factores motivacionales y explorar qué lugar ocupan las noticias falsas en las diferentes dietas. Además, es relevante examinar cómo la exposición a noticias falsas se asocia con la homogeneidad demográfica y sociocultural entre personas conectadas a las redes sociales, así como con características generadas por usuarios en línea (por ejemplo, comentarios de usuarios) y el rol de los algoritmos en la llamada exposición preseleccionada a noticias -incluyendo la exposición a fact-checkers-.

Otro hallazgo relevante es que la autopercepción de la capacidad de reconocer noticias falsas aumenta con una mayor motivación defensiva y una mayor motivación de precisión. Según la teoría del razonamiento motivado (Kunda, 1990), esto sugiere que ambas motivaciones, basadas en el uso de estrategias cognitivas diferentes, permitiría a los diferentes individuos creer que son capaces de reconocer noticias falsas. En el caso de las personas motivadas defensivamente, podrían utilizar la etiqueta de noticia falsa para calificar noticias contra-actitudinales como falsas. Ahora bien, desde la dimensión de género pseudo-periodístico planteada por Egelhofer y Lecheler (2019), es también posible que estas personas podrían identificar noticias falsas -independientemente de si son pro o contra-actitudinalesporque la fortaleza de sus opiniones y actitudes deseadas sobre un tema los haría creer que manejan y tienen certeza de cuál información es veraz. En el caso de las personas motivadas por precisión, las estrategias cognitivas de procesamiento objetivo e imparcial, dirigidas a alcanzar una conclusión correcta sobre un tema, les permitiría identificar falsedades en contenidos noticiosos.

Se encontró que los factores motivacionales se asocian con la exposición y el reconocimiento de noticias falsas, pero no motivan a las personas a difundirlas. A manera hipotética, en el caso de las personas motivadas defensivamente, la meta motivacional se limitaría a catalogar información como falsa para reforzar sus conclusiones deseadas. Estas personas no buscarían una validación social de sus conclusiones mediante la difusión de información falsa, o estarían motivadas a compartir información falsa para intentar influenciar a otras personas. Para las personas motivadas por la precisión, la búsqueda de conclusiones correctas les permite detectar noticias falsas, pero no necesariamente para advertir a otras personas.

En respuesta a la tercera pregunta de investigación, sobre la relación entre las mediciones de noticias falsas y características demográficas, se encontró que las mujeres dicen estar más expuestas a noticias falsas en redes sociales, mientras que los hombres dicen tener más habilidades para reconocerlas. No se observan diferencias según sexo en la exposición a noticias falsas en medios de comunicación y vía WhatsApp. Es posible que estos resultados se asocien con la construcción 
cultural y la socialización del género, que puede reforzar un uso e interpretación diferencial de las noticias falsas, su exposición y la autoeficacia percibida en su reconocimiento. Los antecedentes no nos permiten sustentar las implicancias del hallazgo, por lo que se requiere mayor investigación sobre el tema.

Con respecto al nivel educativo, se encontró que las personas con mayor nivel dicen estar expuestas a noticias falsas y se autoperciben más capaces para reconocerlas. Este hallazgo puede ser resultado de una mayor frecuencia general de consumo noticioso por parte de las personas más educadas y no necesariamente de una mayor habilidad para detectar y rechazar noticias falsas, como Rampersad y Althiyabi (2020) afirmaron en otro contexto para los sectores más educados.

Con respecto a la edad, las personas mayores dicen estar menos expuestas a noticias falsas. No obstante, esto puede deberse a una frecuencia menor de consumo de las fuentes noticiosas estudiadas. Según Rampersad y Althiyabi (2020), las personas de mayor edad aceptan más las noticias falsas, mientras que en Costa Rica se reporta una menor exposición. Sería importante estudiar de forma directa la asociación entre ambos aspectos.

\section{CONCLUSIONES, LIMITACIONES Y FUTURAS INVESTIGACIONES}

¿Cuáles son las implicancias de nuestros hallazgos para la ciudadanía en una sociedad de la desinformación? Primero, nuestros resultados sugieren que la difusión intencional de desinformación puede ser más prevalente entre individuos con actitudes autoritarias. Segundo, las motivaciones que influyen en las estrategias cognitivas para seleccionar y procesar información desempeñan un rol importante en la exposición y en la capacidad autopercibida de reconocer noticias falsas, pero no así en su difusión. Finalmente, para comprender mejor dicha exposición y capacidad de detección de la desinformación, es importante considerar las diferencias sociodemográficas entre ciudadanos/as.

Este estudio no está exento de limitaciones. En primer lugar, desconocemos qué entienden las personas encuestadas por noticia falsa. Como se mencionó, la conceptualización de noticias falsas ha sido objeto de intenso debate académico y su definición es también un producto de su momento histórico. Es posible que las personas encuestadas incluyan acepciones sobre noticias falsas que la literatura académica diferencia. Para atenuar el posible efecto de esta flexibilidad interpretativa, se optó por formular una pregunta en la encuesta que enfatizara la veracidad percibida en la noticia.

En segundo lugar, se realizó un estudio correlacional. Se requieren más investigaciones para establecer relaciones causales entre variables. Sin embargo, 
consideramos que el uso de una muestra representativa a nivel nacional permitió identificar parámetros que aplican a la sociedad costarricense. Otra limitación es la baja confiabilidad de las medidas de need to evaluate y need for cognition, por lo cual se debe considerar que las asociaciones encontradas podrían haber sido más robustas, con medidas más consistentes. Como respuesta a esta limitación psicométrica, futuros estudios pueden utilizar la medición original de need to evaluate que consta de 16 ítems (Jarvis et al., 1996) y la escala original de 18 ítems de need for cognition (Cacioppo \& Petty, 1982) -o bien validar la versión reducida de seis ítems desarrollada por Lins de Holanda Coelho y sus colegas (2018)-.

A pesar de estas precauciones, esta investigación abre valiosas oportunidades para futuros estudios. Una primera línea de trabajo se centra en aspectos de exposición a noticias falsas. Estudios futuros podrían especificar las razones que llevan a las audiencias noticiosas a catalogar un contenido específico como falso. Por ejemplo, es posible que las personas lleguen a esa conclusión debido a una evaluación del contenido de la noticia, porque usan el término de noticia falsa como una etiqueta para darle sentido, o porque la noticia viene acompañada de un comentario que le asigna ese significado de falsedad (tal como un sitio de fact-checking).

También se hace evidente la necesidad de estudios adicionales sobre la difusión de noticias falsas. Por ejemplo, aunque ha sido relativamente posible recopilar datos sobre los hábitos de consumo noticioso en plataformas como Facebook, el estudio de WhatsApp se dificulta por los retos tecnológicos y éticos que plantea. Sin embargo, múltiples investigaciones demuestran su importancia creciente en el consumo de noticias (incluidas las falsas) en la actualidad. Por su parte, este estudio enfatizó la importancia de comprender mejor por qué razones se difunden noticias que se consideran falsas o contra-actitudinales. Todavía es poco claro si se difunden más noticias pro-actitudinales que contra-actitudinales y qué razones podrían explicar estas diferencias. Asimismo, es necesario estudiar variables mediadoras de la influencia del autoritarismo de derecha en la difusión intencional de noticias falsas. Finalmente, es relevante considerar la influencia de variables asociadas con la pertenencia e identificación con endogrupos en la exposición, capacidad de detección y difusión de noticias falsas en redes sociales y WhatsApp en futuras investigaciones.

\section{REFERENCIAS}

Adorno, T., Frenkel-Brunswik, E., Levinson, D., \& Sanford, R. (1950). The authoritarian personality. New York, NY: Harper \& Row.

Altemeyer, B. (1981). Right-wing authoritarianism. Winniped, Canada: University of Manitoba Press.

Altemeyer, B. (1996). The authoritarian specter. Cambridge, MA: Harvard University Press. 
American National Election Studies (2013). American National Election Studies (ANES) Internet Recontact Study. Ann Arbor, MI: Inter-university Consortium for Political and Social Research.

Beck, J. (2019, December 11). This article won't change your mind: The fact on why facts alone can't fight false beliefs. The Atlantic. Retrieved from https://www.theatlantic.com/ science/archive/2017/03/this-article-won't-change-your-mind/519093/

Berinsky, A. A. J. (2017). Rumors and health care reform: Experiments in political misinformation. BritishJournal of Political Science, 47(2), 241-246. https://doi.org//10.1017/S0007123415000186

Bilewicz, M., Soral, W., Marchlewska, M., \& Winiewski, M. (2017). When authoritarians confront prejudice. Differential effects of SDO and RWA on support for hate-speech prohibition. Political Psychology, 38(1), 87-99. https://doi.org/10.1111/pops.12313

Bizer, G., Krosnick, J., Holbrook, A., Petty, R., Rucker, D., \& Wheeler, C. (2002). The Impact of Personality on Political Beliefs, Attitudes, and Behavior: Need for Cognition and Need to Evaluate. In presentation at the Annual Meeting of the American Political Science Association, Boston, MA, August 29-September 1.

Bizer, G., Krosnick, J., Holbrook, A., S. Wheeler, C., Rucker, D., \& Petty, R. (2004). The Impact of Personality on Cognitive, Behavioral, and Affective Political Processes: The Effects of the Need to Evaluate. Journal of Personality, 72(5), 995-1027. https://doi.org/10.1111/j.0022-3506.2004.00288.x

Bronstein, M. V., Pennycook, G., Bear, A., Rand, D. G., \& Cannon, T. D. (2019). Belief in fake news is associated with delusionality, dogmatism, religious fundamentalism, and reduced analytic thinking. Journal of Applied Research in Memory and Cognition, 8(1), 108-117. https://doi.org/10.1016/j.jarmac.2018.09.005

Cacioppo, J. \& Petty, R. (1982). The Need for Cognition. Journal of Personality and Social Psychology, 42(1), 116-131. https://doi.org/10.1037/0022-3514.42.1.116

Cacioppo, J., Petty, R., Feinstein, J., \& Jarvis, B. (1996). Dispositional Differences in Cognitive Motivation: The Life and Times of Individuals Varying in Need for Cognition. Psychological Bulletin, 119(2), 197-253. https://doi.org/10.1037/0033-2909.119.2.197

Carazo, C., Tristán, L., \& Siles, I. (2020). Noticias falsas en Costa Rica: hacia una agenda de investigación (Fake News in Costa Rica: Towards a Research Agenda). In paper presented as a research base for the Informe del Estado de Nación en Desarrollo Humano Sostenible 2020 (Report of the State of the Nation on Sustainable Human Development 2020) (n²6, chapter 11). Retrieved from http://hdl.handle.net/20.500.12337/8025

Crowson, H. M. \& Brandes, J. A. (2017). Differentiating Between Donald Trump and Hillary Clinton Voters Using Facets of Right-Wing Authoritarianism and Social-Dominance Orientation: A Brief Report. Psychological Reports, 120(3), 364-373. https://doi.org/10.1177/0033294117697089

da Silva, A. J. B. \& Larkins, E. R. (2019). The Bolsonaro Election, Antiblackness, and Changing Race Relations in Brazil. The Journal of Latin American and Caribbean Anthropology, 24(4), 893-913. https://doi.org/10.1111/jlca.12438

De keersmaecker, J. \& Roets, A. (2017). 'Fake news': Incorrect, but hard to correct. The role of cognitive ability on the impact of false information on social impressions. Intelligence, 65 , 107-110. https://doi.org/10.1016/j.intell.2017.10.005 
De keersmaecker, J. \& Roets, A. (2019). Is there an ideological asymmetry in the moral approval of spreading misinformation by politicians? Personality and Individual Differences, 143, 165-169. https://doi.org/10.1016/j.paid.2019.02.003

Diakopoulos, N. (2019). Automating the news: How algorithms are rewriting the media. Cambridge, MA: Harvard University Press.

Egelhofer, J. \& Lecheler, S. (2019). Fake news as a two-dimensional phenomenon: a framework and research agenda. Annals of the International Communication Association, 43(2), 97-116. https://doi.org/10.1080/23808985.2019.1602782

Federico, C. \& Schneider, M. (2007). Political Expertise and the Use of Ideology: Moderating Effects of Evaluative Motivation. Public Opinion Quarterly, 71(2), 221-252. https://doi.org/10.1093/poq/nfm010

Guess, A., Nagler, J., \& Tucker, J. (2019). Less than you think: Prevalence and predictors of fake news dissemination on Facebook. Science Advances, 5, eaau4586. https://doi.org/10.1126/sciadv.aau4586

Hameleers, M. \& van der Meer, T. G. (2019). Misinformation and Polarization in a High-Choice Media Environment: How Effective Are Political Fact-Checkers? Communication Research, 47(2), 227-250. https://doi.org/10.1177/0093650218819671

Hansen, G. J. \& Kim, H. (2011). Is the media biased against me? A meta-analysis of the hostile media effect research. Communication Research Reports, 28(2), 169-179. https://doi.org/10.1080/08824096.2011.565280

Hoffmann, S., Taylor, E., \& Bradshaw, S. (2019). The Market of Disinformation. Oxford: Oxford Information Labs. Retrieved from https://oxtec.oii.ox.ac.uk/wp-content/uploads/ sites/115/2019/10/OxTEC-The-Market-of-Disinformation.pdf

Instituto Nacional de Estadística y Censos. (2017). Encuesta Nacional de Hogares 2017 (National Household Survey 2017). San José, Costa Rica: INEC. Retrieved from https://www.inec.cr/sites/default/files/documetos-biblioteca-virtual/reenaho2017.pdf

Ituassu, A., Capone, A., Firmino, L., Magalhães, L., Mannheimer, V., \& Murta, F. (2019). Comunicación política, elecciones y democracia: las campañas de Donald Trump y Jair Bolsonaro (Political Communication, Elections, and Democracy: The Campaigns Of Donald Trump and Jair Bolsonaro). Perspectivas de la comunicación, 12(2), 11-37. https://doi.org/10.4067/S0718-48672019000200011

Jarvis, W., Blair, G., \& Petty, R. (1996). The Need to Evaluate. Journal of Personality and Social Psychology, $70(1), 172-94$. https://doi.org/10.1037/0022-3514.70.1.172

Kahne, J. \& Bowyer, B. (2017). Educating for democracy in a partisan age: Confronting the challenges of motivated reasoning and misinformation. American Educational Research Journal, 54(1), 3-34. https://doi.org/10.3102/0002831216679817

Kunda, Z. (1990). The case for motivated reasoning. Psychological Bulletin, 108(3), 480-498. https://doi.org/10.1037/0033-2909.108.3.480

Lazer, D., Baum, M., Benkler, J., Berinsky, A., Greenhill, K., Metzger, M., \& Zittrain, J. (2018). The science of fake news. Science, 359(6380), 1094-1096. https://doi.org/10.1126/science.aao2998 
Leeper, T. J. \& Slothuus, R. (2014). Political parties, motivated reasoning, and public opinion formation. Political Psychology, 35(S1), 129-156. https://doi.org/10.1111/pops.12164

Lins de Holanda Coelho, G., HP Hanel, P., \& J. Wolf, L. (2018). The very efficient assessment of need for cognition: Developing a six-item version. Assessment, 27(8), 1870-1885. https://doi.org/10.1177/1073191118793208

Montero, J., Zmerli, S., \& Newton, K. (2008). Social trust, political confidence, and satisfaction with democracy. Revista Española DeInvestigaciones Sociológicas (Reis), 122(1), 11-54. Retrieved from https://www.ingentaconnect.com/content/cis/reis/2008/00000122/00000001/art00001

Mustafaraj, E. \& Metaxas, P. T. (2017, June). The fake news spreading plague: Was it preventable? In Proceedings of the 2017 ACM on web science conference (pp. 235-239). https://doi.org/10.1145/3091478.3091523

Newman, N., Fletcher, R., Kalogeropoulos, A., \& Nielsen, R. (2019). Reuters Institute Digital News Report 2019. Oxford, United Kingdom: Reuters Institute for the Study of Journalism.

Nir, L. (2011). Motivated reasoning and public opinion perception. Public Opinion Quarterly, 75(3), 504-532. https://doi.org/10.1093/poq/nfq076

Pérez Sánchez, R., Molina Delgado, M., Smith Castro, V., \& Vega Jiménez, R. (2020). Conservadurismo político e intención de voto durante las elecciones 2017-2018: Una aproximación desde la psicología social (Political conservatism and vote intention during the 2017-2018 elections: An approach from social psychology). In R. A. Redondo \& F. Alpizar Rodríguez (Eds.), Elecciones Costa Rica 2018. Retrato de una Democracia Amenazada (Costa Rica Elections 2018. Portrait of a Threatened Democracy). San José, Costa Rica: Conare.

Pratto, F., Sidanius, J., Stallworth, L. M., \& Malle, B. F. (1994). Social dominance orientation: A personality variable predicting social and political attitudes. Journal of personality and social psychology, 67(4), 741-763. https://doi.org/10.1037/0022-3514.67.4.741

Rampersad, G. \& Althiyabi, T. (2020). Fake news: Acceptance by demographics and culture on social media. Journal of Information Technology \& Politics, 17(1), 1-11. https://doi.org/10.1080/19331681.2019.1686676

Sidanius, J. \& Pratto, F. (1999). Social dominance: An intergroup theory of social hierarchy and oppression. Cambridge, United Kingdom: Cambridge University Press.

Sidanius, J., Pratto, F., Van Laar, C., \& Levin, S. (2004). Social dominance theory: Its agenda and method. Political Psychology, 25(6), 845-880. https://doi.org/10.1111/j.1467-9221.2004.00401.x

Siles, I., Tristán, L., \& Carazo, C. (2021). Populism, media and misinformation in Latin America. In H. Tumber \& S. Waisbord (Eds.), The Routledge companion to media misinformation and populism (pp. 356-365). Routledge.

Sindermann, C., Elhaib, J., Moshagen, M., \& Montag, Ch. (2020): Age, gender, personality, ideological attitudes and individual differences in a person's news spectrum: How many and who might be prone to "filter bubbles" and "echo chambers" online? Heliyon, 6(1), e03214. https://doi.org/10.1016/j.heliyon.2020.e03214

Slater, M. D. (2004). Operationalizing and analyzing exposure: The foundation of media effects research. Journalism \& Mass Communication Quarterly, 81(1), 168-183.

https://doi.org/10.1177/107769900408100112 
Taber, C. S. \& Lodge, M. (2006). Motivated skepticism in the evaluation of political beliefs. American Journal of Political Science, 50(3), 755-769. https://doi.org/10.1111/j.1540-5907.2006.00214.x

Taber, C. S., Cann, D., \& Kucsova, S. (2009). The motivated processing of political arguments. Political Behavior, 31, 137-155. https://doi.org/10.1007/s11109-008-9075-8

Tandoc, E. C. J., Lim, Z. W., \& Ling, R. (2018). Defining “fake news”. Digital Journalism, 6(2), 137-153. https://doi.org/10.1080/21670811.2017.1360143

Tandoc, E. C., Ling, R., Westlund, O., Duffy, A., Goh, D., \& Zheng Wei, L. (2018). Audiences' acts of authentication in the age of fake news: A conceptual framework. New Media $\mathcal{E}$ Society, 20(8), 2745-2763. https://doi.org/10.1177/1461444817731756

Tong, C., Gill, H., Li, J., Valenzuela, S., \& Rojas, H. (2020). “Fake News Is Anything They Say!"-Conceptualization and Weaponization of Fake News among the American Public. Mass Communication and Society,23(5), 755-778. https://doi.org/10.1080/15205436.2020.1789661

Valenzuela, S., Bachmann, I., \& Bargsted, M. (2021). The personal is the political? What do Whatsapp users share and how it matters for news knowledge, polarization and participation in Chile. Digital Journalism, 9(2), 155-175. https://doi.org/10.1080/21670811.2019.1693904

van der Linden, S., C. Panagopoulos, \& Roozenbeek, J. (2020). You Are Fake News: Political Bias in Perceptions of Fake News. Media, Culture \& Society, 42 (3), 460-470. https://doi.org/10.1177/0163443720906992

Wagner, M. C. \& Boczkowski, P. J. (2019). The Reception of Fake News: The Interpretations and Practices That Shape the Consumption of Perceived Misinformation. Digital Journalism, 7(7), 870-885. https://doi.org/10.1080/21670811.2019.1653208

Waisbord, S. (2018). Truth is what happens to news: On journalism, fake news, and posttruth.Journalism Studies, 19(13), 1866-1878. https://doi.org/10.1080/1461670X.2018.1492881

Winter, S., Metzger, M. J., \& Flanagin, A. J. (2016). Selective Use of News Cues: A MultipleMotive Perspective on Information Selection in Social Media Environments. Journal of Communication, 66(4), 669-693. https://doi.org/10.1111/jcom.12241 


\section{FINANCIAMIENTO}

Nuestra investigación no recibió financiamiento de ningún organismo. La Rectoría de la Universidad de Costa Rica donó los fondos para realizar el trabajo de campo.

\section{SOBRE LOS AUTORES}

CARLOS BRENES PERALTA, investigador. Labora en el Instituto de Investigaciones Psicológicas de la Universidad de Costa Rica. Realizó sus estudios de posgrado en Investigación Social y Comunicación en la Universidad Libre de Amsterdam (Holanda) y su doctorado en Comunicación Política en la Universidad de Amsterdam. Áreas de interés investigativo: la psicología social de los usos y efectos de las tecnologías de la información y la comunicación, la exposición selectiva y procesamiento de contenido noticioso, la investigación en opinión pública, desinformación y noticias falsas.

(iD) https://orcid.org/0000-0003-0578-3908

ROLANDO PÉREZ SÁNCHEZ, Profesor catedrático. Universidad de Costa Rica. Labora en el Instituto de Investigaciones Psicológicas y en la Escuela de Psicología de la Universidad de Costa Rica. Sus estudios doctorales los realizó en Sociología de la Comunicación en la Universidad de Frankfurt (Alemania). Áreas de interés investigativo: la psicología social de los usos y efectos de las tecnologías de la información y la comunicación, así como los procesos socio-cognitivos y emocionales asociados al uso de redes sociales.

iD https://orcid.org/0000-0001-6321-2543

IGNACIO SILES GONZÁLEZ, Profesor Catedrático. Labora en el Centro de Investigación en Comunicación (CICOM) y en la Escuela de Ciencias de la Comunicación Colectiva de la Universidad de Costa Rica. Realizó estudios de doctorado en Medios, Tecnología y Sociedad en la Universidad Northwestern (Estados Unidos). Áreas de interés investigativo: comunicación, tecnología y sociedad; estudios críticos de la datificación. 